\title{
The role of NbTMP1, a surface protein of sporoplasm, in Nosema bombycis infection
}

\author{
Shiyi Zheng 1,2,4 Yukang Huang ${ }^{1,2}$, Hongyun Huang ${ }^{1,2}$, Bin Yu ${ }^{1,2}$, Ni Zhou ${ }^{1,2}$, Junhong Wei ${ }^{1,2}$, Guoqing Pan ${ }^{1,2}$,
} Chunfeng $\mathrm{Li}^{1,2^{*}}$ and Zeyang Zhou ${ }^{1,2,3}$

\begin{abstract}
Background: Nosema bombycis is a unicellular eukaryotic pathogen of the silkworm, Bombyx mori, and is an economic and occupational hazard in the silkworm industry. Because of its long incubation period and horizontal and vertical transmission, it is subject to quarantine measures in sericulture production. The microsporidian life-cycle includes a dormant extracellular phase and intracellular proliferation phase, with the proliferation period being the most active period. This latter period lacks spore wall protection and may be the most susceptible stage for control.

Methods: In order to find suitable target for the selective breeding of N. bombycis-resistant silkworm strains, we screen highly expressed membrane proteins from the transcriptome data of $\mathrm{N}$. bombycis. The subcellular localization of the candidate protein was verified by Indirect immunofluorescence analysis (IFA) and immunoelectron microscopy (IEM), and its role in N. bombycis proliferation was verified by RNAi.

Results: The N. bombycis protein (NBO_76g0014) was identified as a transmembrane protein and named NbTMP1. It is homologous with hypothetical proteins NGRA_1734 from Nosema granulosis. NbTMP1 has a transmembrane region of 23 amino acids at the $\mathrm{N}$-terminus. Indirect immunofluorescence analysis (IFA) results suggest that NbTMP1 is secreted on the plasma membrane as the spores develop. Western blot and qRT-PCR analysis showed that NbTMP1 was expressed in all developmental stages of $\mathrm{N}$. bombycis in infected cells and in the silkworm midgut. Downregulation of NbTMP1 expression resulted in significant inhibition of N. bombycis proliferation.

Conclusions: We confirmed that NbTMP1 is a membrane protein of $\mathrm{N}$. bombycis. Reduction of the transcription level of NbTMP1 significantly inhibited N. bombycis proliferation, and this protein may be a target for the selective breeding of N. bombycis-resistant silkworm strains.
\end{abstract}

Keywords: Nosema bombycis, Membrane protein, Localization, RNAi, Monoclonal antibody

\section{Introduction}

Microsporidia are obligate intracellular parasitic eukaryotes with a wide range of hosts, infecting almost all vertebrates and invertebrates, including humans [1-3]. These spore-forming unicellular parasites have a unique ultrastructure and life-cycle [3]. They were

*Correspondence: licf@swu.edu.cn

1 State Key Laboratory of Silkworm Genome Biology, Southwest University, Chongqing 400715, China

Full list of author information is available at the end of the article originally considered to be primitive organisms, such as protozoans or protists [4]. However, microsporidia are now considered to be related to fungi or perhaps to be a sister branch of fungi that has lost many genes and undergone genome compression, possibly due to their adaptation to intracellular parasitism [5-8]. The lack of mitochondria is taken as evidence that microsporidia are ancient eukaryotes [9]. As a result of the loss of many metabolic pathways, microsporidia must obtain nutrients from the host and export the cytotoxic compounds through the cell membrane [10-12]. original author(s) and the source, provide a link to the Creative Commons licence, and indicate if changes were made. The images or other third party material in this article are included in the article's Creative Commons licence, unless indicated otherwise in a credit line to the material. If material is not included in the article's Creative Commons licence and your intended use is not permitted by statutory regulation or exceeds the permitted use, you will need to obtain permission directly from the copyright holder. To view a copy of this licence, visit http://creativecommons.org/licenses/by/4.0/. The Creative Commons Public Domain Dedication waiver (http://creativeco mmons.org/publicdomain/zero/1.0/) applies to the data made available in this article, unless otherwise stated in a credit line to the data. 
Microsporidia have a distinctive mechanism for infecting host cells. The polar tube is ejected when microsporidia are stimulated by suitable conditions, and the infective sporoplasm is transferred into the host cells through the polar tube prior to proliferation [13]. The sporoplasm can also adhere to host cells and enter the host cells by phagocytosis [14]. The membrane proteins of the sporoplasm then interact with the cytoplasma content of host cell.

Nosema bombycis, the first microsporidian to be recognized, by Nageli in 1857, causes silkworm pébrine disease by vertical and horizontal transmission [15]. The prevention and control of $N$. bombycis is an important focus of sericulture research. In this study, we identified a transmembrane protein (NBO_76g0014) of N. bombycis, which we designated NbTMP1. We cloned and expressed recombinant protein NbTMP1 and prepared monoclonal antibodies. We also characterized the subcellular localization of NbTMP1 in N. bombycis and explored its function in $N$. bombycis proliferation.

\section{Materials and methods}

Preparation of $\mathbf{N}$. bombycis and cell cultivation

Mature spores of N. bombycis CQ1, obtained from the China Veterinary Culture Collection Center (CVCC No. 102059), were isolated from infected silkworm pupae and purified by Percoll density gradient centrifugation $(21,000 \mathrm{~g}, 40 \mathrm{~min})$ [16]. Cells of ovarian cell line Spodoptera frugiperda (Sf9-III) in Sf-900 III $^{\mathrm{TM}}$ SFM were purchased from Thermo Fisher Scientific (Waltham, MA, USA) and cultured in Sf- $900^{\mathrm{TM}}$ III SFM medium at $28^{\circ} \mathrm{C}$.

\section{NbTMP1 sequence analysis and amplification of the open reading frame}

NbTMP1 (GenBank Accession No. EOB13409.1), which is a highly expressed protein in the early stages of infection, was screened from the transcriptome data of Bombyx mori infected with $N$. bombycis. The amino acid sequence of NbTMP1 was submitted to the SignalP 5.1 server (http://www.cbs.dtu.dk/services/SignalP/) and the TMHMM server v.2.0 (http://www.cbs.dtu.dk/ services/TMHMM/) for signal peptide and transmembrane domain predictions. The molecular weight and isoelectric point ( $\mathrm{pI}$ ) were predicted using the ExPASy server (http://web.expasy.org/compute_pi/), and the protein function domain was predicted using SMART (http://smart.embl-heidelberg.de/). BLAST (https:// blast.ncbi.nlm.nih.gov/) was used to analyze the multiple sequence alignment. We cloned the extramembranous region of $N b T M P 1$ using the forward primer
5'-GGATCCATGTTCAAGTCTAGTGATGA-3' containing a $B a m H I$ restriction site (underlined bases) and the reverse primer $5^{\prime}$-GTCGACCTTATCATT TTCATTATTTCCC-3' $3^{\prime}$ containing a Sall restriction site (underlined bases). Complementary DNA (cDNA) was obtained by reverse transcription of RNA from the Sf9-III cells (Thermo Fisher Scientific) infected with $N$. bombycis and used as the PCR template. The PCR products were purified with the Gel Extraction Kit of Omega Bio-tek, Inc. (Norcross, GA, USA) and integrated into the pET-28 vector, following which the vectors were transformed into competent cells of Escherichia coli strain DH5 $\alpha$. The positive pET-28NbTMP1 recombinant vectors were sequenced by Sangon (Shanghai, China).

\section{Recombinant protein expression, monoclonal antibody preparation and immunoblotting}

The identified pET-28-NbTMP1 vector was transformed into E. coli strain Rosetta for expression. The recombinant bacteria were induced for $4 \mathrm{~h}$ at $37{ }^{\circ} \mathrm{C}$ with $0.5 \mathrm{mM}$ isopropyl- $\beta$-D-thiogalactopyranoside (IPTG) in LB medium. The target protein was purified with nickel-chelating affinity chromatography (Roche Applied Science, Penzberg, Germany). All animal experiments were approved by the Laboratory Animals Ethics Review Committee of Southwest University (Chongqing, China) under Permit Number AERCSWU2017-7. The mice were maintained according to the recommendations of the committee, and food and water were provided ad libitum. Female BALB/c mice (6- to 8 weeks-old) were injected subcutaneously four times with rNbTMP1 (100 $\mu \mathrm{g} /$ mouse $)$ mixed with Freund's complete/incomplete adjuvant (1:1; SigmaAldrich, St. Louis, MO, USA). The injection interval was 7 days. Monoclonal antibodies (mAbs) were produced and screened as previously described [17]. The $\mathrm{mAb}$ immunoglobin G (IgG) subtypes were detected using the Mouse Monoclonal Antibody Isotyping Kit (Roche Applied Science) according to manufacturer's recommendations.

For immunoblotting, the proteins of mature spores and infected cells were prepared by the glass bead breaking method [18], isolated by sodium dodecyl sulfate-polyacrylamide gel elctrophoresis and transferred to PDVF membranes (Roche Applied Science). After blocking in $5 \%$ skim milk diluted in TBST $(150 \mathrm{mM} \mathrm{NaCl}, 20 \mathrm{mM}$ Tris- $\mathrm{HCl}, 0.05 \%$ Tween-20), the membrane was incubated for $1 \mathrm{~h}$ in anti-NbTMP1 ascites (1:1000). After washing three times, the membrane was incubated with 
goat anti-mouse IgG (1:5000; Sigma-Aldrich) conjugated with horseradish peroxidase (HRP) label. Finally, the protein bands were visualized with ECL Plus Western Blotting Detection Reagents (Bio-Rad, Hercules, CA, USA).

\section{Silkworm midgut paraffin section}

Newly molted fifth instar silkworm larvae were starved for 1 day and then fed mulberry leaves coated with spores $\left(1 \times 10^{7}\right.$ spores per larva). At 4 days post-infection (dpi), the infected silkworms were killed, and the midguts were collected and stored at $-80^{\circ} \mathrm{C}$. For embedding, the midguts were first washed three times, 15 min each time, in phosphate buffered saline (PBS: $137 \mathrm{mM} \mathrm{NaCl}, 10 \mathrm{mM}$ $\mathrm{Na}_{2} \mathrm{HPO}_{4}, 2 \mathrm{mM} \mathrm{KH_{2 }} \mathrm{PO}_{4}$ and $2.7 \mathrm{mM} \mathrm{KCl}$ ), following which the midguts were fixed in $4 \%$ paraformaldehyde for $24 \mathrm{~h}$. The sections were prepared as previously described [19]. After deparaffination, the sections were boiled in sodium citrate buffer solution for $10 \mathrm{~min}$ and then used in the immunolocalization studies.

\section{Immunofluorescence analysis}

For the immunofluorescence analysis (IFA), the samples were fixed in $4 \%$ paraformaldehyde and then washed three times in PBS. The samples were then permeabilized with $0.5 \%$ Triton X-100 for $30 \mathrm{~min}$ and then blocked with PBS-bovine serum albumin for $1 \mathrm{~h}$ at room temperature. Next, the samples were incubated with the primary antibodies, including anti-NbTMP1 ascites (mouse), or with negative serum and $\mathrm{Nb} \beta$-tubulin (rabbit) antibody which was used to label meronts of N. bombycis [20]. After washing three times with PBS, Alexa 488 conjugate Goat anti-Mouse IgG and Alexa 568 conjugate Goat anti-Rabbit IgG (Thermo Fisher Scientific) were used to detect the bound primary antibodies, and 4',6-diamidino-2-phenylindole (DAPI) (Thermo Fisher Scientific) was used to label the nucleus. The results were observed under a confocal laser scanning microscope (Olympus, Tokyo, Japan).

\section{Immunoelectron microscopy study}

Infected silkworm midguts were used for the immunoelectron microscopy (IEM) study. Ultrathin sections $(70 \mathrm{~nm})$ were prepared as previously described [19] and placed on nickel grids. After blocking with PBS-bovine serum albumin, the grids were incubated with antiNbTMP1 ascites diluted 1:30 or with negative serum at room temperature for $1 \mathrm{~h}$. The grids were then incubated with gold-conjugated anti-mouse IgG (Aldrich-Sigma) diluted to 1:100. After staining with uranium and lead, the sections were examined and photographed with a JEM-1400 Plus TEM transmission electron microscope (JEOL Co. Ltd., Tokyo, Japan).

\section{RNA interference of NbTMP1}

A 331-bp interferential fragment was selected by BLOCK-iT ${ }^{\mathrm{TM}}$ RNAi Designer (http://rnaidesigner.therm ofisher.com/rnaiexpress/design.do). The fragment was amplified by the F-RI-NbTMP1-T7 (5'-TAATACGAC TCACTATAGGGAGATGTCTCTAGGGCAGATGC-3') and R-RI-NbTMP1-T7 (5'-TAATACGACTCACTATAG GGAGATCCAGTACGTGTTGCCTG-3') primers and used as the template for synthesizing double-stranded RNA (dsRNA). The RiboMAX ${ }^{\mathrm{TM}}$ Large Scale RNA Production System-T7 (Promega, Madison, WI, USA) was used to obtain the dsRNA. The dsRNA of enhanced green fluorescent protein (EGFP) was used as a negative control and was synthesized as described above [17]. After the Sf9-III cells were cultured in 12-well plates overnight, $2 \mu \mathrm{g}$ dsRNA of NbTMP1 or EGFP was transfected into the cells. After $4 \mathrm{~h}$, the spores were germinated with 0.1 $\mathrm{M} \mathrm{KOH}$ and added to the Sf9-III cells (spores:cell, 5:1). Samples of infected cells were collected at 1,3 , and $5 \mathrm{dpi}$ and immediately stored in PBS or TRIzol (Invitrogen, Carlsbad, CA, USA) at $-80^{\circ} \mathrm{C}$.

\section{Real-time quantitative PCR analysis}

A DNA Extraction Kit (Omega Bio-tek, Inc.) was used to extract the genomic DNA (gDNA) of the infected cells. Total RNA Kit II (Omega Bio-tek, Inc.) and EvoScript Universal cDNA Master (Roche Applied Science) were used to prepare the cDNA. Real-time quantitative PCR (qPCR) was amplified by the F-q-Nb TMP1 (5'-CCT ATCTCTAAAGACGGT-3') and R-q-Nb TMP1 (5'-CTT TTTCTATTTTGGCAGCA-3') primers, and reference gene primers $\mathrm{F}$-q-SSU (5'-CTGGGGATAGTATGATCG CAAGA-3') and R-q-SSU (5'-CACAGCATCCATTGG AAACG-3'). The transcription levels were calculated by the $2^{-\triangle \triangle t}$ values method with three replicates. GraphPad Prism v6.01 (GraphPad Software, San Diego, CA, USA) was used to conduct the multiple $t$ tests.

The NbB-tubulin copy number was used to count $N$. bombycis. The gDNA of infected cells samples was analyzed by qPCR. The reaction systems were conducted using the primers: $\mathrm{Nb} \beta$-tubulin-qF (5'-AGAACCAGG AACAATGGACG- $3^{\prime}$ ) and Nb $\beta$-tubulin-qR (5'-AGC CCAATTATTACCAGCACC-3'). The standard template used was described in previous research [17]. The standard curve covered six orders of magnitude $(1.3 \times$ $\left.10^{2}-10^{7}\right)$.

\section{Results}

Sequence characteristics and immunoblot analysis of NbTMP1

Due to the loss of many metabolic pathways, microsporidia must obtain nutrients and energy from the host through the cell membrane. In order to facilitate 
the control of $N$. bombycis in the cell, we attempted to screen highly expressed membrane proteins from the transcriptome data of $N$. bombycis. NbTMP1, a hypothetical membrane protein, was highly expressed in the transcriptome data of Bombyx mori infected with $N$. bombycis, suggesting that it may be involved in the proliferation of $N$. bombycis. Sequence analysis showed that NbTMP1 is $43 \%$ homologous to the hypothetical protein NGRA_1734 (GenBank Accession No. KAF9762805.1) in Nosema granulosis [21]. NbTMP1 contained a complete open reading frame (ORF) that was $732 \mathrm{bp}$ in length and encoded a polypeptide of 243 amino acids with a transmembrane domain. The protein has a calculated molecular weight of $27.59 \mathrm{kDa}$ and a theoretical pI value of 9.92. It does not have a signal peptide and typical functional domains. The recombinant NbTMP1 protein was expressed as a soluble protein of about $30 \mathrm{kDa}$ (Fig. 1a) and was purified to prepare the monoclonal antibody F12.

Western blot analysis with F12 ascites revealed a unique positive band in mature spores and in the proteins of infected cells (Fig. 1b). The band is about $35 \mathrm{kDa}$, which is more than the molecular weight of NbTMP1, possibly due to post-translational modification, such as phosphorylation and glycosylation, of the native NbTMP1. Bioinformatics analysis showed that there are two glycosylation sites and 20 phosphorylation sites. Since the recombinant protein lacks a transmembrane domain, the molecular mass of the recombinant NbTMP1 and the native protein differed. The Mouse Monoclonal Antibody Isotyping Kit (Roche Applied Science) was used to identify the subtypes of monoclonal antibody F12, and the test strip demonstrated that the subtypes of F12 were IgG1-k (Fig. 1c).

\section{Subcellular localization of NbTMP1}

To study the subcellular localization of NbTMP1, we performed IFA with the F12 ascites. Sporoplasm was obtained by allowing mature spores to germinate in 0.1 $\mathrm{M} \mathrm{KOH}$. The IFA indicated that NbTMP1 is localized on the plasma membrane of the sporoplasm (Fig. 2). We used the $\mathrm{Nb} \beta$-tubulin antibody to label meronts and noted that NbTMP1 co-located with $\mathrm{Nb} \beta$-tubulin in the proliferation stage (Fig. 3). The subcellular localization of NbTMP1 was verified by examing paraffin sections of infected tissues. The fluorescence signal of NbTMP1 was consistent with the contour of mature spores (Fig. 4a), but there was no fluorescence signal in mature spores that had not been sectioned (Fig. 4b). As antibodies cannot penetrate the spore wall of $N$. bombycis, this

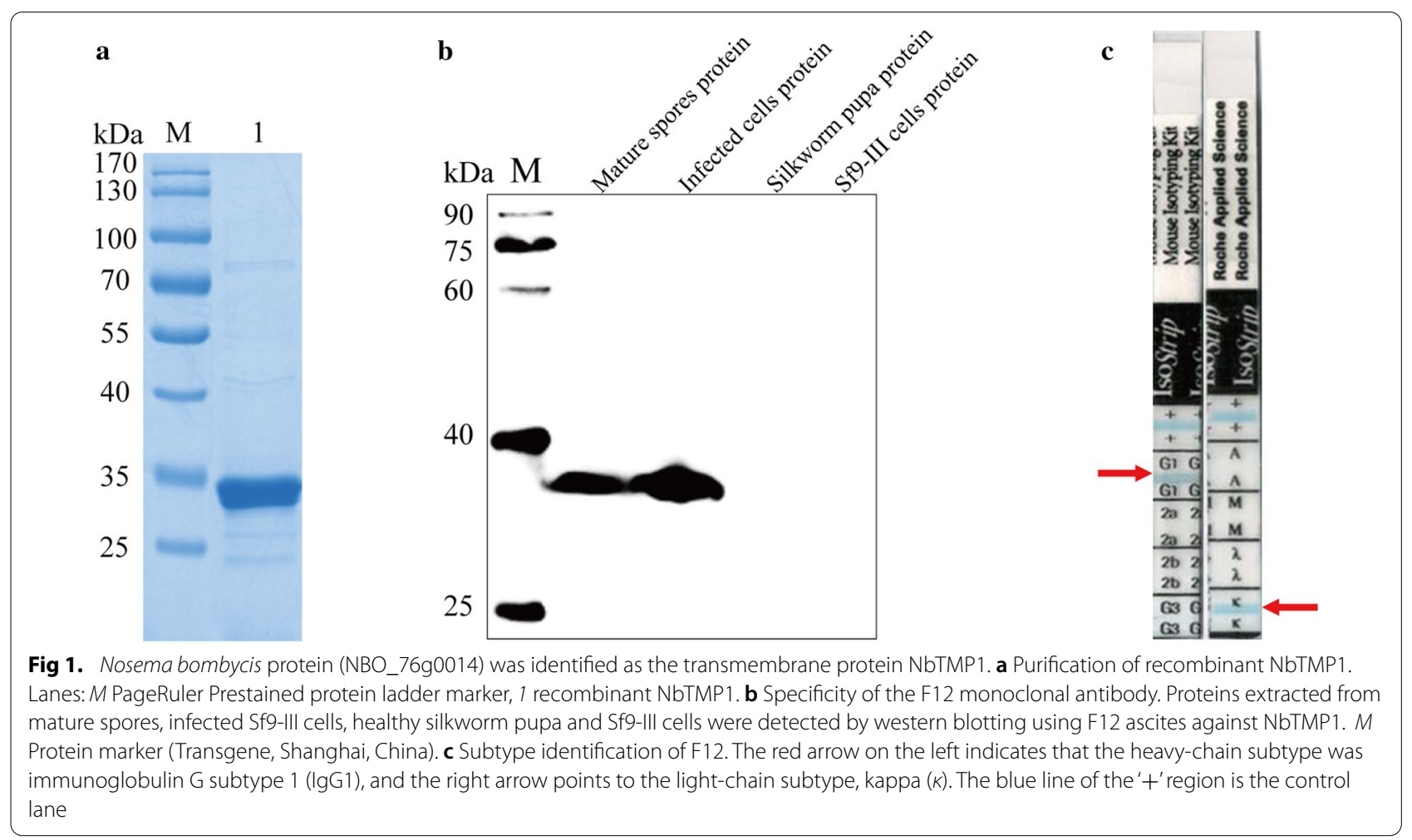


observation also implies that NbTMP1 is located on the plasma membrane. IEM analysis further demonstrated that NbTMP1 is located on the membrane of N. bombycis (Fig. 5). This result indicates that NbTMP1 may be secreted to the plasma membrane during the development of N. bombycis.

\section{Transcriptional profile of NbTMP1 in infected cells and midguts}

After $N$. bombycis infected the Sf9-III cells, the expression of NbTMP1 increased from the 1 dpi to $6 \mathrm{dpi}$. The drop in NbTMP1 expression on 4 dpi may be due to the formation of mature spores (Fig. 6a). In the midguts
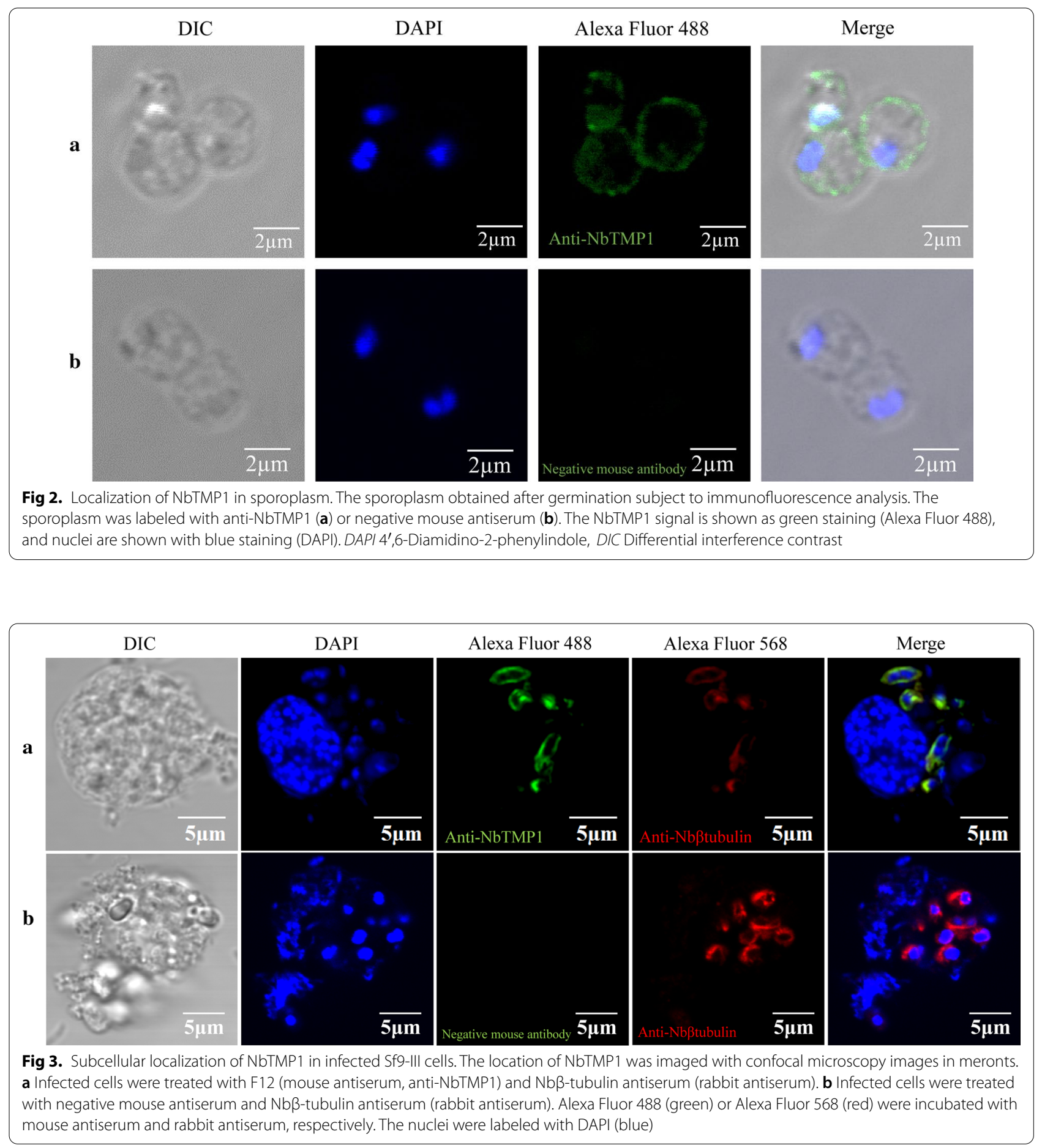


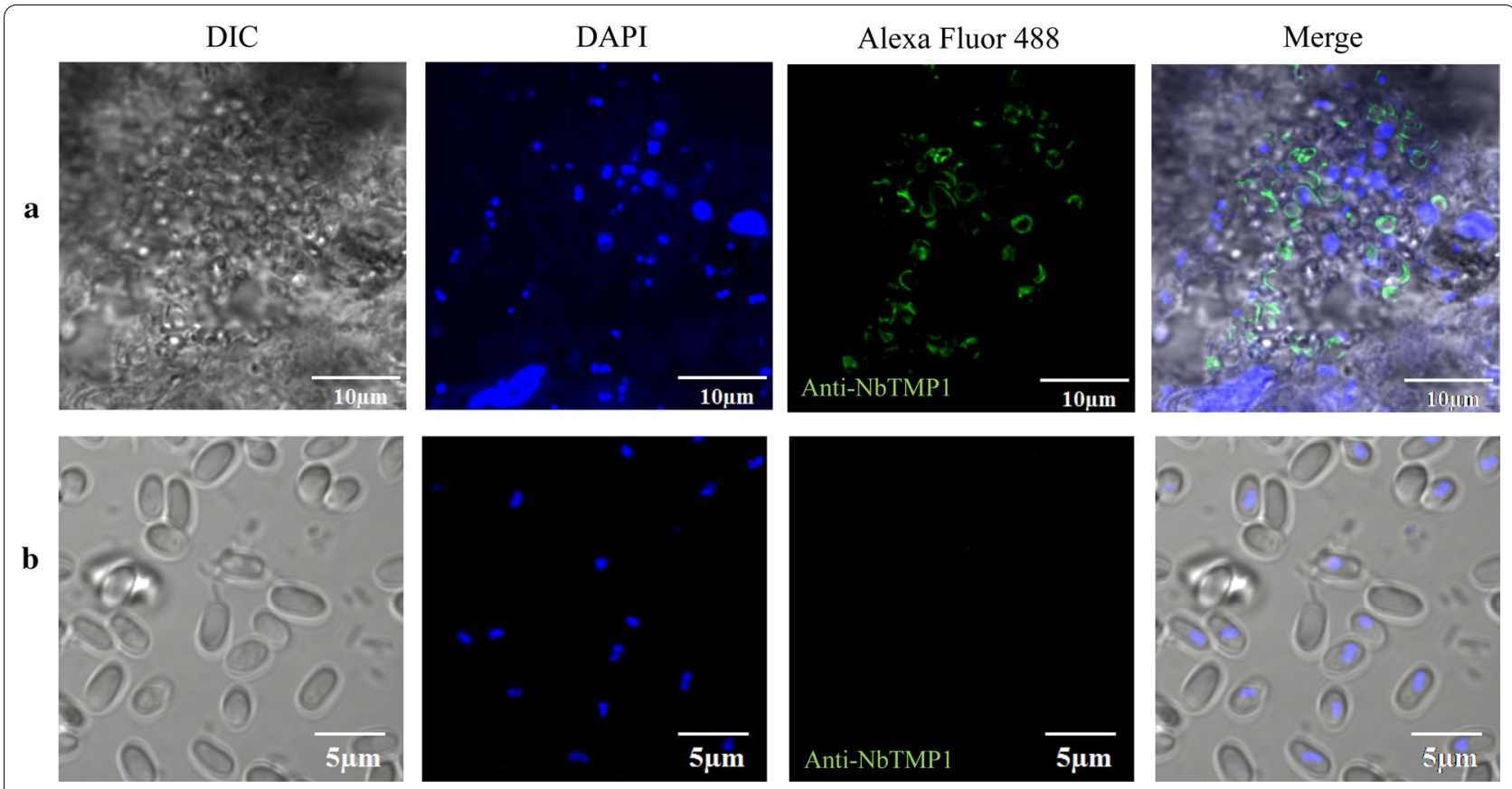

Fig 4. Subcellular localization of NbTMP1 in infected silkworm midguts (a) and mature spores (b). The midgut tissue section and unsliced mature spores were labeled with DAPI (nuclei) and anti-NbTMP1 (green)

of infected silkworms, NbTMP1 showed low expression level in the pre-infection phase, with expression increasing continuously after 4 dpi (Fig. 6b). On 4 dpi, $N$. bombycis began a new round of host infection, and the transcript profile of NbTMP1 suggested that it may play a role in spore infection.

\section{RNA interference of NbTMP1 inhibited N. bombycis' proliferation}

The effects of RNA interference (RNAi) were analyzed using qPCR. Following the addition of the dsRNA, the transcription level of NbTMP1 was significantly downregulated in the experimental groups (NbTMP1-dsRNA) (Fig. 7a). Since Nbß-tubulin is a housekeeping and conserved gene of $N$. bombycis, its copy number was used to reflect the number of $N$. bombycis in the two groups [17]. The qPCR results showed that $N$. bombycis began to proliferate from 1 to $5 \mathrm{dpi}$ in the mock groups (EGFPdsRNA). However, the pathogen load was remarkably lower in the experimental groups (Fig. 7b). These results suggest that NbTMP1 plays an important role in the proliferation of $N$. bombycis.

\section{Discussion}

Membrane protein is rarely studied in microsporidia. In Trachipleistophora hominis, plasma membrane-located purine nucleotide transport proteins (NTTs) are key components of the process involved in stealing ATP from hosts [11]. EhSSP1 was identified as Encephalitozoon hellem sporoplasm surface protein 1; this protein binds to human foreskin fibroblasts and is associated with the final step of invasion on the invasion synapse [22]. NbTMP1 was predicted to have a transmembrane domain, suggesting that it may be located on the membrane of the spore. The IFA and IEM results proved that NbTMP1 actually did localize on the membrane of sporoplasm and spores. The expression profiles in vitro and in vivo and the western blot results showed that NbTMP1 is present in all stages of the $N$. bombycis life-cycle. Although we showed that downregulation of NbTMP1 expression can inhibit the proliferation of $N$. bombycis, the specific mechanism remains unknown.

There are two main hypotheses on how microsporidia infect host cells. One is that the microsporidia eject a polar tube, penetrate the membrane of new host cells and then deliver the contents into the cytoplasm of the host cell; the other is that the microsporidia gain access to the host cell by phagocytosis, and then the polar tube is used to escape the phagosome and to infect the host cytoplasm [13]. Sporoplasm is the earliest stage of microsporidia infection of host cells. The mechanism used by microsporidia to transmit genetic information after infecting a host cell is unclear. However, surface proteins, such as NbAQP, NbSWP9 and NbSWP7, can play important roles in the process of spore germination and infection [23, 24]. NbTMP1 is located on the 

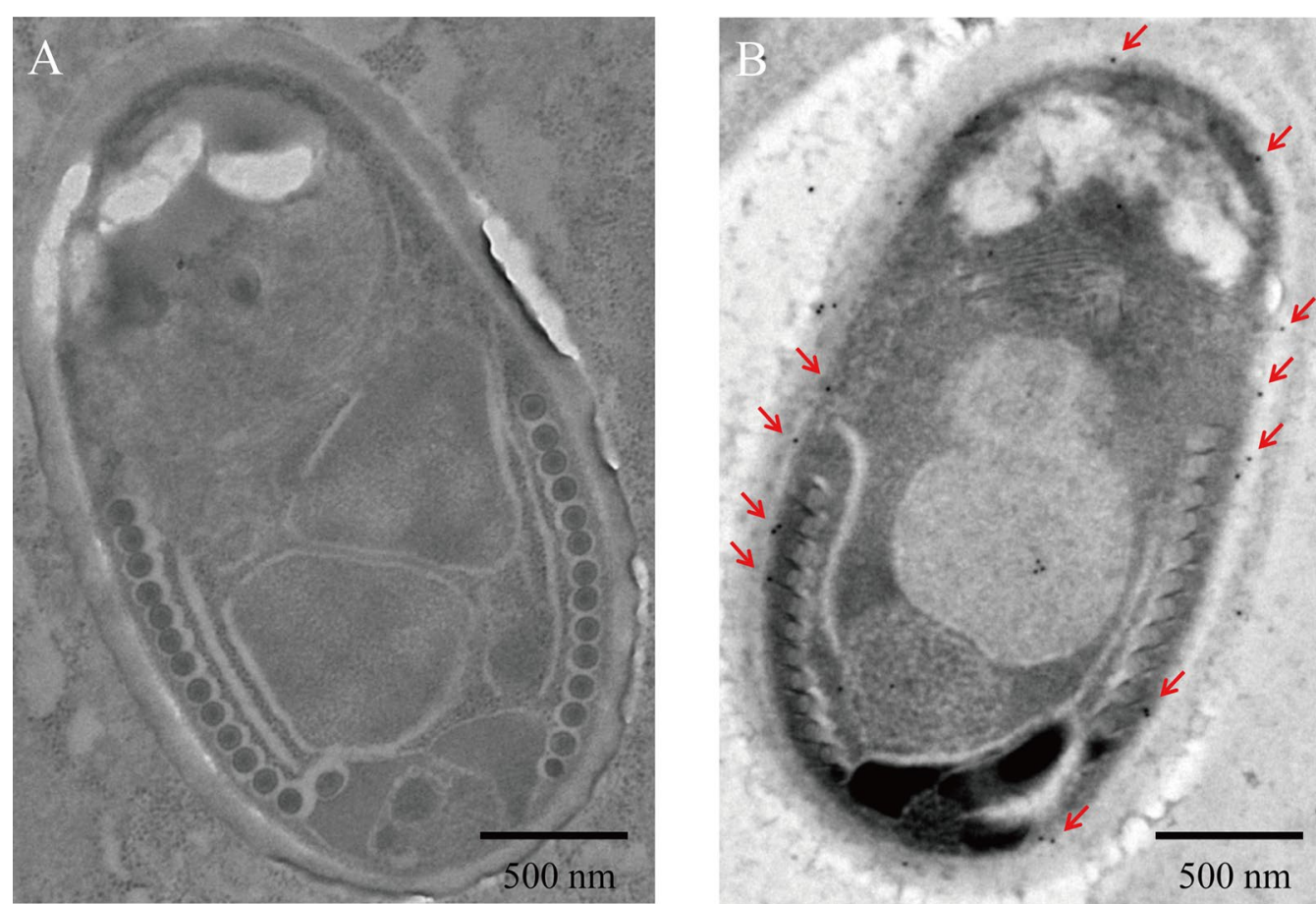

Fig 5. Immunoelectron microscopy of NbTMP1 in spores of infected midgut. a Negative control, b spore showing the presence of gold labeled anti-NbTMP1 on the plasma membrane. The red arrow indicates the gold label. Scale bars $500 \mathrm{~nm}$
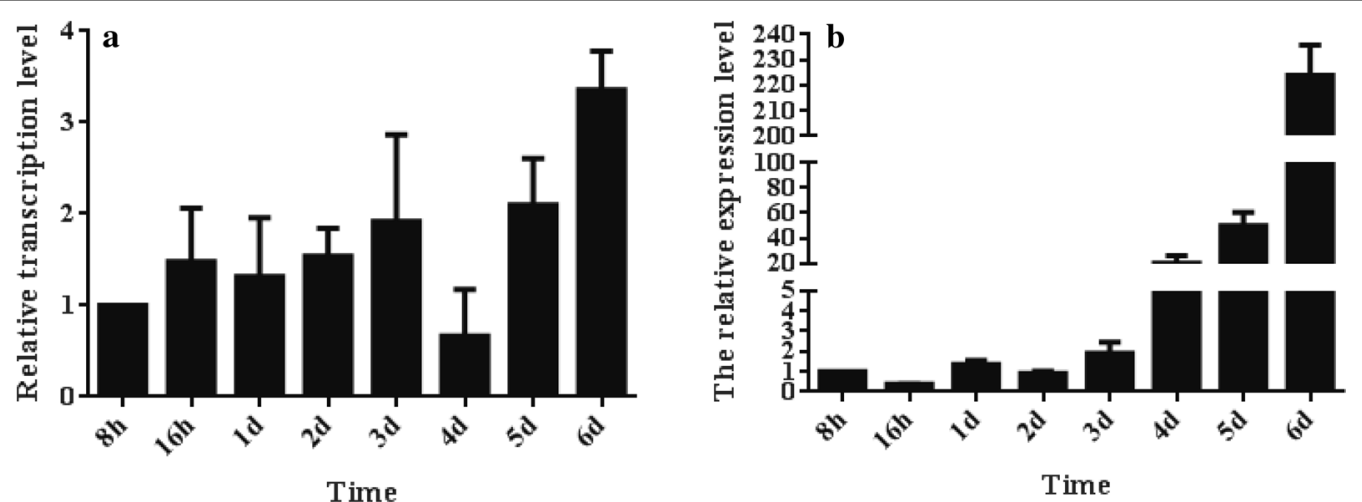

Fig 6. Transcription analysis of NbTMP1. N. bombycis small subunit ribosomal ribonucleic acid was used as an internal control. The relative expression level of NbTMP1 is presented relative to expression at $8 \mathrm{~h}$ post-infection. a Transcription level of NbTMP1 in infected Sf9-III cells, $\mathbf{b}$ transcription level of NbTMP1 in infected silkworm midguts. Vertical bars show the mean \pm standard error $(n=3)$

plasma membrane of the sporoplasm, suggesting that it may be involved in the proliferation of spores in cells.

Understanding the protein functions of $N$. bombycis has been hampered by the lack of stable and reliable gene manipulation methods. RNAi has been used in fungi, including Heterosporis saurida and Nosema ceranae, to study protein functions $[25,26]$. Nosema bombycis possesses all of the genes required for RNA silencing and in vivo function [27]. A stable RNAi strategy was previously established utilizing an in vitro transcription system and lipofection in Sf9-III infection [28]. RNAi has been successfully used to develop Bombyx mori nucleopolyhedrovirus (anti-BmNPV)resistant silkworms [29]. RNAi can also be used as a strategy for breeding $N$. bombycis-resistant silkworms, and NbTMP1 is a potential target of RNAi. Single-chain 


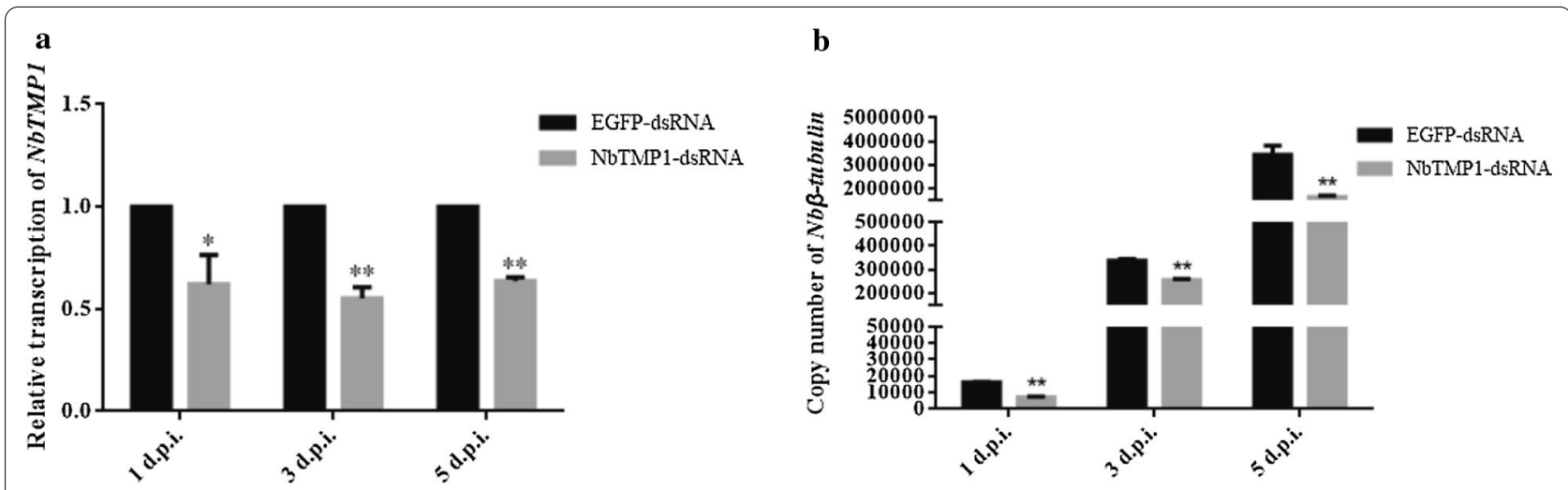

Fig 7. Effect of downregulated NbTMP1 on N. bombycis proliferation via RNA interference. a The transcription levels of NbTMP1 in infected Sf9-III cells with NbTMP1-dsRNA or EGFP-dsRNA at 1, 3 and 5 days post infection (d.p.i.). b The number of N. bombycis in Sf9-III cells. The copy number of $\mathrm{Nb} \beta$-tubulin indicates the number of $\mathrm{N}$. bombycis. Asterisks represent statistically significant differences at ${ }^{*} P<0.05$ and $\left.{ }^{* *} P<0.01\right)$. Scale bars represent the standard deviation of three independent repeats. EGFP Enhanced green fluorescent protein

antibodies are also a strategy for breeding $N$. bombycis-resistant silkworms. In Anopheles, transposonmediated transformation was used to generate $\mathrm{m} 1 \mathrm{C} 3$, m4B7 and m2A10 single-chain antibodies (scFvs), and the transgenic mosquitoes expressing the scFv gene had significantly lower infection levels of Plasmodium falciparum [30, 31]. The transgenic Sf9-III cells, which express single-chain antibodies of SWP12 of N. bombycis, effectively inhibit the proliferation of $N$. bombycis in cells [17]. In this study, we obtained the monoclonal antibody (F12) of NbTMP1, and in future investigations we plan to evaluate the performance of this singlechain antibody for breeding of $N$. bombycis-resistant silkworms. In follow-up studies, we will try to express dsRNA of NbTMP1 and scFv-F12 in silkworm individuals by transgenic technology to verify the function of NbTMP1 and evaluate the resistance of transgenic silkworms to $N$. bombycis.

\section{Conclusions}

We identified a novel membrane protein in the microsporidium $N$. bombycis. NbTMP1 was localized on the plasma membrane of the sporoplasm, meronts and spores, and its proliferation was significantly inhibited by RNAi. This membrane localized protein is important for pathogen development, and may be a potential target for construction of $N$. bombycis-resistant silkworms.

\section{Abbreviations}

IFA: Indirect immunofluorescence analysis; IEM: Immunoelectron microscopy; Sf9-III cells: Ovarian cell line of Spodoptera frugiperda; RNAi: RNA interference; qPCR: Real-time quantitative PCR.

\section{Acknowledgements}

We thank our colleagues Dr. Xianzhi Meng for his help in the the IEM experiment and Dr. Qiang He for his guidance on sporoplasm separation. We also thank LetPub (www.letpub.com) for its linguistic assistance during the preparation of this manuscript.

\section{Authors' contributions}

$\mathrm{SZ}, \mathrm{YH}$ and $\mathrm{HH}$ performed the exeriments and analyzed the data. SZ and $\mathrm{CL}$ drafted the manuscript and performed manuscript preparation. $\mathrm{SZ}, \mathrm{HH}, \mathrm{BY}$ and $N Z$ supervised and performed the collection of material and all other laboratory experiments. SZ, JW, GP, CL and ZZ conceived the idea and coordinated the project. All authors read and approved the final manuscript.

\section{Funding}

This work was supported by the National Natural Science Foundation of China (Grant number: 31770160, 31702185,32070795) and Fundamental Research Funds for the Central Universities to Southwest University (XDJK2018AA001).

\section{Availability of data and materials}

Data supporting the conclusions of this article are included within the article. All data are fully available without restriction upon reasonable request.

\section{Ethics approval and consent to participate}

All animal procedures were approved by Laboratory Animals Ethics Review Committee of Southwest University (Chongqing, China) under Permit Number: AERCSWU2017-7.

\section{Consent for publication}

Not applicable.

\section{Competing interests}

The authors declare that they have no competing interests.

\section{Author details}

'State Key Laboratory of Silkworm Genome Biology, Southwest University, Chongqing 400715 , China. ${ }^{2}$ Chongqing Key Laboratory of Microsporidia Infection and Control, Southwest University, Chongqing 400715, China. ${ }^{3}$ College of Life Sciences, Chongqing Normal University, Chongqing 401331, China. ${ }^{4}$ Affiliated Jinhua Hospital, Zhejiang University of Medicine-Jinhua Municipal Central Hospital, Jinhua 321000, Zhejiang, China.

Received: 27 November 2020 Accepted: 11 January 2021

Published online: 25 January 2021 


\section{References}

1. Han B, Polonais V, Sugi T, Yakubu R, Takvorian PM, Cali A, et al. The role of microsporidian polar tube protein 4 (PTP4) in host cell infection. PLoS Pathog. 2017;13(4):e1006341.

2. Didier ES, Didier PJ, Snowden KF, Shadduck JA. Microsporidiosis in mammals. Microbes Infect. 2000;2(6):709-20.

3. Cali A, Takvorian PM. Developmental morphology and life cycles of the microsporidia. Hoboken: John Wiley \& Sons, Ltd; 1999.

4. Vossbrinck CR, Maddox JV, Friedman S, Debrunner-Vossbrinck BA, Woese CR. Ribosomal RNA sequence suggests microsporidia are extremely ancient eukaryotes. Nature. 1987;326(6111):411-4.

5. Keeling PJ, Mcfadden Gl. Origins of microsporidia. Trends Microbiol. 1998:6(1):19.

6. Thomarat F, Vivares C, Gouy M. Phylogenetic analysis of the complete genome sequence of Encephalitozoon cuniculi supports the fungal origin of microsporidia and reveals a high frequency of fast-evolving genes. J Mol Evol. 2004;59(6):780-91.

7. Corradi N. Microsporidia: eukaryotic intracellular parasites shaped by gene loss and horizontal gene transfers. Annu Rev Microbiol. 2015;69(69):167.

8. Capella-Gutiérrez S, Marcet-Houben M, Gabaldón T. Phylogenomics supports microsporidia as the earliest diverging clade of sequenced fungi. BMC Biol. 2012;10:47.

9. Hacker C, Howell M, Bhella D, Lucocq J. Strategies for maximizing ATP supply in the microsporidian Encephalitozoon cuniculi: direct binding of mitochondria to the parasitophorous vacuole and clustering of the mitochondrial porin VDAC. Cell Microbiol. 2014;16(4):565-79.

10. Cuomo CA, Desjardins CA, Bakowski MA, Jonathan G, Ma AT, Becnel JJ, et al. Microsporidian genome analysis reveals evolutionary strategies for obligate intracellular growth. Genome Res. 2012;22(12):2478-88.

11. Eva H, Christian H, Paul D, John M, Goldberg AV, Williams TA, et al. Plasma membrane-located purine nucleotide transport proteins are key components for host exploitation by microsporidian intracellular parasites. PLoS Pathog. 2014;10(12):e1004547.

12. Heinz E, Williams TA, Nakjang S, Noël CJ, Swan DC, Goldberg AV, et al. The genome of the obligate intracellular parasite Trachipleistophora hominis: new insights into microsporidian genome dynamics and reductive evolution. PLoS Pathog. 2012;8(10):e1002979.

13. Franzen C. Microsporidia: how can they invade other cells? Trends in parasitology. 2004;20(6):275-9.

14. He Q, Luo J, Xu JZ, Wang CX, Meng XZ, Pan GQ, et al. Morphology and transcriptome analysis of Nosema bombycis sporoplasm and insights into the initial infection of microsporidia. mSphere. 2020;5(1):e00958-e1019.

15. Han MS, Watanabe $H$. Transovarial transmission of two microsporidia in the silkworm, Bombyx mori, and disease occurrence in the progeny population. J Invertebr Pathol. 1988;51(1):41-5.

16. Wu Z, Li Y, Pan G, Tan X, Xiang Z. Proteomic analysis of spore wall proteins and identification of two spore wall proteins from Nosema bombycis (Microsporidia). Proteomics. 2010;8(12):2447-61.

17. Huang Y, Chen J, Sun B, Zheng R, Li B, Li Z, et al. Engineered resistance to Nosema bombycis by in vitro expression of a single-chain antibody in Sf9-III cells. PLoS One. 2018;13(2):e0193065
18. Zhi L, Pan G, Tian L, Wei H, Jie C, Geng L, et al. SWP5, a spore wall protein, interacts with polar tube proteins in the parasitic microsporidian Nosema bombycis. Eukaryot Cell. 2012;11(2):229-37.

19. Meng $X Z$, Luo $B$, Tang $X Y$, , He Q, Xiong TR, Fang ZY, et al. Pathological analysis of silkworm infected by two microsporidia Nosema bombycis CQ1 and Vairimorpha necatrix BM. J Invertebr Pathol. 2018;153:75-84.

20. Chen J, Guo W, Dang X, Huang Y, Liu F, Meng X, et al. Easy labeling of proliferative phase and sporogonic phase of microsporidia Nosema bombycis in host cells. PLoS One. 2017;12(6):e0179618.

21. Cormier A, Chebbi MA, Giraud I, Wattier R, Teixeira M, Gilbert C, et al. Comparative genomics of strictly vertically transmitted, feminizing microsporidia endosymbionts of amphipod crustaceans. Genome Biol Evol. 2020. https://doi.org/10.1093/gbe/evaa245.

22. Han B, Ma Y, Tu V , Tomita T. Microsporidia interact with host cell mitochondria via voltage-dependent anion channels using sporoplasm surface protein 1. mBio. 2019;10(4):e01944-e2019.

23. Chen G, Wang W, Chen H, et al. Functional characterization of an aquaporin from a microsporidium, Nosema bombycis. PLoS One. 2017;12(7):e181703

24. Yang D, Pan G, Dang X, Shi Y, Li C, Peng P, et al. Interaction and assembly of two novel proteins in the spore wall of the microsporidian species Nosema bombycis and their roles in adherence to and infection of host cells. Infect Immun. 2015;83(4):1715-31.

25. Nitzan P, Eitan G, Maayan O, Yaron Z, Lucie A, Jeffery P, et al. Effective gene silencing in a microsporidian parasite associated with honeybee (Apis mellifera) colony declines. Appl Environ Microbiol. 2010;76(17):5960-4

26. Saleh M, Kumar G, Abdel-Baki AA, Dkhil MA, El-Matbouli M, Al-Quraishy S. In vitro gene silencing of the fish microsporidian Heterosporis saurida by RNA interference. Nucleic Acid Ther. 2016;26(4):250-6.

27. Pan Q, Wang L, Dang X, Ma Z, Zhang X, Chen S, et al. Bacterium-expressed dsRNA downregulates microsporidia Nosema bombycis gene expression. J Eukaryot Microbiol. 2017;64(2):278.

28. Huang Y, Zheng S, Mei X, Yu B, Sun B, Li B, et al. A secretory hexokinase plays an active role in the proliferation of Nosema bombycis. Peer J. 2018;6:e5658.

29. Kanginakudru S, Royer C, Edupalli SV, Jalabert A, Mauchamp B, et al. Targeting ie-1 gene by RNAi induces baculoviral resistance in lepidopteran cell lines and in transgenic silkworms. Insect Mol Biol. 2010;16(5):635-44.

30. Isaacs AT, Fengwu L, Nijole J, Xiaoguang C, Xavier N, Osvaldo M, et al. Engineered resistance to Plasmodium falciparum development in transgenic Anopheles stephensi. PLoS Pathog. 2011;7(4):e1002017.

31. Isaacs AT, Nijole J, Mikhail T, Isabelle T, Agnès Z, Catherine $B$, et al. Transgenic Anopheles stephensi coexpressing single-chain antibodies resist Plasmodium falciparum development. Proc Natl Acad Sci USA 2012:109(28):11070-1.

\section{Publisher's Note}

Springer Nature remains neutral with regard to jurisdictional claims in published maps and institutional affiliations.

Ready to submit your research? Choose BMC and benefit from

- fast, convenient online submission

- thorough peer review by experienced researchers in your field

- rapid publication on acceptance

- support for research data, including large and complex data types

- gold Open Access which fosters wider collaboration and increased citations

- maximum visibility for your research: over 100M website views per year

At BMC, research is always in progress.

Learn more biomedcentral.com/submissions 